\title{
Marketing odontológico: análise de anúncios publicitários de acordo com código de ética
}

\author{
Dental marketing: analysis of advertising according of ethical code
}

\author{
Tiago Ribeiro Leal ${ }^{1}$, Herdesson Pereira Silva ${ }^{1}$, Lorenna Mendes Temóteo Brandt ${ }^{2}$, Alessandro Leite \\ Cavalcanti $^{3 *}$ \\ ${ }^{1}$ Acadêmico do Curso de Graduação em Odontologia. Universidade Estadual da Paraíba (UEPB); ${ }^{2}$ Mestre em \\ Odontologia pelo Programa de Pós-Graduação em Odontologia. Universidade Estadual da Paraíba (UEPB); \\ ${ }^{3}$ Professor Doutor do Programa de Pós-Graduação em Odontologia. Universidade Estadual da Paraíba (UEPB)
}

\begin{abstract}
Resumo
Objetivo: avaliar os aspectos éticos relacionados à propaganda e publicidade veiculadas pelos cirurgiões-dentistas. Metodologia: estudo transversal que avaliou os anúncios publicitários de 40 consultórios e 17 clínicas odontológicas da cidade de Campina Grande, PB. Os anúncios foram analisados de acordo com o tipo de publicidade, com a especialidade apresentada pelo profissional ou clínica, com a apresentação de itens obrigatórios, itens permitidos e itens proibidos segundo as normas éticas da Resolução CFO118/2012. Os dados coletados foram registrados em formulário específico, inseridos em banco de dados do SPSS, versão 18.0 e apresentados por meio da estatística descritiva. Resultados: verificou-se que o meio de comunicação mais usado pelos profissionais da Odontologia é a placa, com $84,2 \%$. Dentre os anúncios analisados, apenas $12,3 \%$ apresentavam todos os itens obrigatórios exigidos pelo Código de Ética Odontológica. O nome representativo da profissão "cirurgião-dentista" esteve presente em apenas 14,0\% dos estabelecimentos pesquisados. Dentre os itens permitidos, informações sobre a área de atuação foram verificadas em $54,4 \%$ dos anúncios; entretanto, somente 3,5\% dos anúncios apresentavam o título de especialidade. Quanto às infrações éticas, 5,3\% dos anúncios apresentaram elementos proibidos pelo Código de Ética Odontológica, como imagens de "antes e depois" e modalidade de pagamento. Conclusões: os profissionais não estão respeitando as normas estabelecidas pelo Conselho Federal de Odontologia no que concerne à propaganda e publicidade.
\end{abstract}

Palavras-chave: Legislação Odontológica. Marketing de Serviços Odontológicos. Código de Ética.

\begin{abstract}
Objective: the aim of this study was to evaluate ethical features concerning to advertising and publicity of dental establishments. Methodology: this cross-sectional study analyzed forty dental offices and seventeen dental clinics situated in Campina Grande, PB. Dental advertisements were surveyed according to the kind of publicity, dental specialty, mandatory items, elective items and banned items, according to CFO-118/2012 rule. Data were recorded in a specific form, entered into SPSS 18.0 database and presented using descriptive statistics. Results: the kind of publicity most prevalent on $84.2 \%$ dentistry establishments was signboard. Among analyzed announcements, just 12.3\% show all mandatory items according Dental Code of Professional Conduct. The expression "cirurgiãodentista" (dentist) was present in a few $14.0 \%$ of surveyed ads. In case of elective items, occupation area informations were present in $54.4 \%$ of establishments, however only $3.5 \%$ of signboards present specialist titles. With regard to ethical violation, $5.3 \%$ of publicity exhibit some banned item, such as "before and after" and payment method. Conclusions: dentists are not complying rules preestablished by the Federal Council of Dentistry regarding the advertising and publicity.
\end{abstract}

Keywords: Legislation. Dental. Marketing of Dental Services. Code of Ethics.

\section{INTRODUÇÃO}

O cenário da Odontologia no Brasil passou por grandes modificações a partir da década de 80 , nos aspectos tecnológico e social. De uma profissão que se estruturava na relação de confiança entre paciente e profissional, o cirurgião-dentista $(C D)$, atualmente, necessita utilizar adequadamente o marketing e a publicidade como meios para atrair pacientes. Algumas das modificações pelas quais passou o mercado de trabalho odontológico podem

Correspondente/Corresponding: * Alessandro Leite Cavalcanti - Departamento de Odontologia Universidade Estadual da Paraíba - UEPB Rua Juvêncio Arruda, s/no - Bodocongó 58.100-000. Campina Grande -PB-Brasil-Tel: (83) 3315-3326-E-mail: dralessandro@ibest.com.br ser atribuídas ao crescimento do número de faculdades de Odontologia (INEP, 2015), que inserem aproximadamente 17.000 profissionais em um mercado que vem apresentando sinais visíveis de saturação (CAVALCANTI et al., 2011; GARBIN et al., 2010; MARTINS et al., 2011).

Nesta perspectiva, ser um bom profissional não é o suficiente para o cirurgião-dentista se destacar em uma área tão competitiva, principalmente nos grandes centros urbanos onde a concentração de profissionais é maior. Noções de administração, gerenciamento e planejamento são indispensáveis para aquele cirurgião-dentista que faz a opção de exercer a Odontologia de forma liberal (DA COSTA, 2015). A competitividade induz o profissional a 
buscar novas estratégias na tentativa de atrair clientes e de formar sua carteira de pacientes (SILVA et al., 2013).

As regras para o exercício da Odontologia estão contidas no Código de Ética Odontológica (CEO), aprovado pela Resolução CFO no118, de 11 de maio de 2012 (BRASIL, 2012), elaborado a partir da IV Conferência Nacional de Ética Odontológica (BENEDICTO et al., 2013). O CEO estabelece os fundamentos básicos que regem a prática do cirurgião-dentista no exercício de sua profissão, conscientizando também sobre a responsabilidade civil que recai sobre o profissional da odontologia (PACHECO; SILVA JÚNIOR; MEIRELES, 2014).

No Capítulo XVI "Do anúncio, da propaganda e da publicidade", que trata exclusivamente das formas de divulgação, consta, em seu Artigo 43, a obrigatoriedade do nome e número de inscrição no Conselho Regional de Odontologia (CRO) de pessoa física ou jurídica juntamente com o nome representativo da profissão cirurgião-dentista. No caso de pessoa jurídica, devem constar, ainda, o nome e a inscrição do responsável técnico (BRASIL, 2012).

Em caráter optativo, segundo o CEO, podem constar áreas de atuação; procedimentos e técnicas de tratamento, desde que precedidos pelo título de especialidade; títulos de formação ou a expressão "clínico geral", caso os conhecimentos tenham sido adquiridos em curso de graduação ou cursos de aprofundamento; endereço; telefone; endereço eletrônico; horário de trabalho; convênio; credenciamento; atendimentos domiciliar e hospitalar; logomarca ou logotipo (BRASIL, 2012).

$O$ atual CEO caracteriza como infrações éticas a propaganda enganosa; a mercantilização da Odontologia; o emprego da expressão "popular"; o oferecimento de gratuidade com intenção de autopromoção; apresentação de imagens que indiquem o "antes e depois" de um procedimento clínico; e outras condutas que sejam relacionadas ao aviltamento da profissão (BRASIL, 2012).

Portanto, é dever do cirurgião-dentista anunciar de maneira clara e ética, promovendo a concorrência de maneira leal, e assumindo postura ética que, indiscutivelmente, contribua não só para a fidelização dos pacientes como para a boa conceituação da profissão perante a sociedade (CAVALCANTI et al., 2011; PARANHOS et al., 2011).

O objetivo deste trabalho foi analisar se os cirurgiões-dentistas atuantes na cidade de Campina Grande - PB, que fazem uso de publicidade em Odontologia por meio de anúncios, respeitam os parâmetros estabelecidos pelo Código de Ética Odontológico.

\section{METODOLOGIA}

O estudo caracterizou-se como sendo transversal, quantitativo e descritivo, utilizando a observação direta como técnica de pesquisa.

A pesquisa foi realizada no município de Campina Grande, PB, situado no agreste paraibano, considerado um dos principais polos industriais da região e um importante centro tecnológico do país. Abrange uma área de 595 km², possui uma população de 405 mil habitantes e IDH de 0,72 (PARAÍBA, 2009; IBGE, 2016). Conforme dados do Conselho Federal de Odontologia, existem 790 cirurgiões-dentistas registrados no município (CFO, 2009,2015).

Para a seleção da amostra, foi solicitada, inicialmente, ao Conselho Regional de Odontologia da Paraíba (CRO-PB) a lista dos profissionais cadastrados e seus respectivos endereços profissionais. Todavia, por restrições legais, não foi permitido o acesso a esses dados. Assim, elegeu-se o website publicitário "TeleListas.net" (TELELISTAS. NET, 2016) como base para a obtenção das informações referentes aos profissionais atuantes no município. $\mathrm{E}$ a amostra, do tipo não-probabilística, foi composta por 40 consultórios e 17 clínicas odontológicas.

Foram excluídos da amostra aqueles consultórios situados em prédios comerciais que impossibilitavam a presença de mídia externa; anúncios afixados em locais de difícil visualização, como os escondidos por árvores ou detalhes arquitetônicos; e anúncios afixados no interior de clínicas; com sinais de rasuras, ilegíveis ou qualquer outro tipo de adulteração.

Para a coleta de dados, utilizou-se um formulário com as seguintes variáveis: região (centro e outros bairros); tipo de publicidade; especialidade apresentada pelo profissional ou clínica; presença de itens obrigatórios (nome do profissional, número de inscrição no CRO-PB e nome representativo da profissão); itens permitidos (telefone, área de atuação, logomarca, endereço, horário de trabalho, especialidade, convênios e títulos de formação) e itens proibidos (imagens de "antes e depois", modalidade de pagamento), segundo as normas éticas do CEO, descritos na Resolução CFO-118/2012 (BRASIL, 2012). A coleta de dados foi realizada por um pesquisador, previamente treinado, que visitou os endereços das clínicas e consultórios e verificou a publicidade in loco, procedendo ao preenchimento dos itens do formulário.

As informações coletadas foram registradas em um banco de dados com o software SPSS 18.0 (Statistical Package for the Social Sciences) e apresentadas por meio da estatística descritiva (distribuição absoluta e percentual).

\section{RESULTADOS}

Verificou-se que 70,2\% dos estabelecimentos pesquisados são consultórios de pequeno porte, enquanto 29,8\% são considerados clínicas localizadas, em sua maioria, $(49,1 \%)$ no centro da cidade. Um expressivo número de estabelecimentos utilizou a placa $(84,2 \%)$ como principal forma de divulgação. Dentre os anúncios analisados, verificou-se a divulgação da área de atuação apenas em $35,1 \%$ dos consultórios e em 19,3\% das clínicas. As especialidades mais prevalentes foram a Reabilitação Oral/ Prótese (33,3\%), Dentística (28,1\%) e Ortodontia (26,3\%). Apenas 3,5\% dos consultórios e clínicas apresentaram informação sobre o título de especialista. 
Tabela 1 - Distribuição do tipo de publicidade, área de atuação e título de especialidade veiculados pelas clínicas e consultórios odontológicos.

\begin{tabular}{|c|c|c|c|c|c|c|}
\hline \multirow[b]{2}{*}{ Variável } & \multicolumn{2}{|c|}{ Consultório } & \multicolumn{2}{|c|}{ Clínica } & \multicolumn{2}{|c|}{ Total } \\
\hline & $\mathbf{n}$ & $\%$ & $\mathbf{N}$ & $\%$ & $\mathbf{n}$ & $\%$ \\
\hline \multicolumn{7}{|l|}{ Tipo de Publicidade* } \\
\hline Placa & 33 & 57,9 & 15 & 26,3 & 48 & 84,2 \\
\hline Adesivo & 4 & 7,0 & 5 & 8,8 & 9 & 15,8 \\
\hline Letreiro (Pintura) & 9 & 15,8 & - & - & 9 & 15,8 \\
\hline Faixa de Pano & 1 & 1,8 & - & - & 1 & 1,8 \\
\hline \multicolumn{7}{|l|}{ Apresenta Área de Atuação } \\
\hline Sim & 20 & 35,1 & 11 & 19,3 & 31 & 54,4 \\
\hline Não & 20 & 35,1 & 6 & 10,5 & 26 & 45,6 \\
\hline \multicolumn{7}{|l|}{ Área de Atuação* } \\
\hline Reabilitação Oral / Prótese & 13 & 22,8 & 6 & 10,6 & 19 & 33,3 \\
\hline Dentística & 9 & 15,8 & 7 & 12,3 & 16 & 28,1 \\
\hline Ortodontia & 7 & 12,3 & 8 & 14,0 & 15 & 26,3 \\
\hline Implantodontia & 7 & 12,3 & 4 & 7,0 & 11 & 19,3 \\
\hline Cirurgia & 5 & 8,8 & 3 & 5,3 & 8 & 14,0 \\
\hline Endodontia & 4 & 7,0 & 4 & 7,0 & 8 & 14,0 \\
\hline Periodontia & 1 & 1,8 & 1 & 1,8 & 2 & 3,5 \\
\hline \multicolumn{7}{|l|}{$\begin{array}{l}\text { Apresenta Título } \\
\text { de Especialista }\end{array}$} \\
\hline Sim & 1 & 1,8 & 1 & 1,8 & 2 & 3,5 \\
\hline Não & 39 & 68,4 & 16 & 28,1 & 55 & 96,5 \\
\hline
\end{tabular}

*Mais de uma opção.

Fonte: Os autores

No que diz respeito aos itens obrigatórios, apenas $12,3 \%$ (7) dos estabelecimentos apresentaram todos os itens de acordo com o CEO. Destes, 71,4\% (5) são consultórios e $28,6 \%$ (2) são clínicas. O nome do profissional foi o item mais prevalente, constando em $54,4 \%$ dos consultórios e em $10,5 \%$ das clínicas. O nome "cirurgião-dentista", representativo da profissão, foi negligenciado por $86,0 \%$ dos estabelecimentos, sendo substituído pela palavra Dentista $(22,4 \%)$; por imagens de dentes ou de instrumentos odontológicos (18,4\%); Odonto (16,3\%); Odontologia (14,3\%); e expressões genéricas que remetam ao tratamento odontológico, tais como: Consultório odontológico (8,2\%), Dental (8,2\%), “Orto" (6,1\%), Aparelho ortodôntico $(4,1 \%)$. Ou, ainda, foi substituído pelo título de especialidade (2,0\%) (Tabela 2$)$.
Tabela 2 - Distribuição dos itens obrigatórios, de acordo com o Código de Ética Odontológico, nos anúncios de propaganda e publicidade veiculados pelas clínicas e consultórios odontológicos.

\begin{tabular}{lcccccc}
\hline Itens Obrigatórios & \multicolumn{3}{c}{ Consultórios } & \multicolumn{2}{c}{ Clínicas } & \multicolumn{2}{c}{ Total } \\
& $\mathbf{n}$ & $\%$ & $\mathbf{N}$ & $\%$ & $\mathbf{n}$ & $\%$ \\
\hline Nome do profissional & & & & & & \\
Sim & 31 & 54,4 & 6 & 10,5 & 37 & 64,9 \\
Não & 9 & 15,8 & 11 & 19,3 & 20 & 35,1 \\
Inscrição no CRO* & & & & & & \\
Sim & 14 & 24,6 & 5 & 8,8 & 19 & 33,3 \\
Não & 26 & 45,6 & 12 & 21,5 & 38 & 66,7 \\
Termo "cirurgião-dentista" & & & & & & \\
Sim & 6 & 10,5 & 2 & 3,5 & 8 & 14,0 \\
Não & 34 & 59,6 & 15 & 26,3 & 49 & 86,0
\end{tabular}

Termos de substituição

\begin{tabular}{lcccccc} 
Dentista & 9 & 18,4 & 2 & 4,1 & 11 & 22,4 \\
Imagem & 5 & 10,2 & 4 & 8,2 & 9 & 18,4 \\
Odonto & 7 & 14,3 & 1 & 2,0 & 8 & 16,3 \\
Odontologia & 4 & 8,2 & 3 & 6,1 & 7 & 14,3 \\
Dental & 2 & 4,1 & 2 & 4,1 & 4 & 8,2 \\
Consultório Odontológico & 4 & 8,2 & - & - & 4 & 8,2 \\
“Orto" & 3 & 6,1 & - & - & 3 & 6,1 \\
Aparelho Ortodôntico & - & - & 2 & 4,1 & 2 & 4,1 \\
Especialidade & - & - & 1 & 2,1 & 1 & 2,1 \\
\hline
\end{tabular}

${ }^{*} \mathrm{CRO}=$ Conselho Regional de Odontologia

Fonte: Os autores

Dos itens opcionais permitidos pelo CEO, o mais prevalente foi a divulgação do telefone $(77,2 \%)$, seguido por área de atuação $(54,4 \%)$, logotipo ou logomarca $(21,1 \%)$ e endereço $(17,5 \%)$ (Tabela 3$)$.

No que se refere aos itens proibidos pelo CEO, verificou-se, em 5,3\% dos anúncios, a presença de um ou mais itens. Observou-se, como infração mais prevalente, a modalidade de pagamento, encontrada em 5,3\% dos estabelecimentos, seguida pelo anúncio de "antes e depois", verificado em 1,8\% desses anúncios. 
Tabela 3 - Distribuição dos itens opcionais e proibidos, de acordo com o Código de Ética Odontológico, nos anúncios de propaganda e publicidade veiculados pelas clínicas e consultórios odontológicos.

\begin{tabular}{lcccccc}
\hline & $\begin{array}{c}\text { Consul- } \\
\text { tórios }\end{array}$ & Clínicas & Total \\
\hline Itens Opcionais & $\mathbf{n}$ & $\%$ & $\mathbf{N}$ & $\%$ & $\mathbf{N}$ & $\%$ \\
Telefone & 27 & 47,4 & 17 & 29,8 & 44 & 77,2 \\
Área de atuação & 20 & 35,1 & 11 & 19,3 & 31 & 54,4 \\
Logomarca & 5 & 8,8 & 7 & 12,3 & 12 & 21,1 \\
Endereço & 8 & 14 & 2 & 3,5 & 10 & 17,5 \\
Horário de trabalho & 4 & 7 & 1 & 1,8 & 5 & 8,8 \\
Especialidade & 1 & 1,8 & 1 & 1,8 & 2 & 3,5 \\
Convênios & 2 & 3,5 & - & - & 2 & 3,5 \\
Títulos de formação & 1 & 1,8 & - & - & 1 & 1,8
\end{tabular}

Itens Proibidos

$\begin{array}{lllllll}\operatorname{Sim} & 1 & 1,8 & 2 & 3,5 & 3 & 5,3\end{array}$

$\begin{array}{lllllll}\text { Não } & 39 & 68,4 & 15 & 26,3 & 54 & 94,7\end{array}$

Tipo de infração

$\begin{array}{llllllll}\text { Antes e Depois } & - & - & 1 & 1,8 & 1 & 1,8\end{array}$

$\begin{array}{lllllll}\text { Modalidade de pagamento } & 1 & 1,8 & 2 & 3,5 & 3 & 5,3\end{array}$

*CRO = Conselho Regional de Odontologia

Fonte: Os autores

Dos estabelecimentos pesquisados, apenas $12,3 \%$ estavam totalmente de acordo com a normatização estabelecida pelo CEO. Entre estes, 05 (cinco) consultórios e 02 (duas) clínicas apresentaram todos os itens obrigatórios e não incorrendo em nenhum tipo de infração ética.

\section{DISCUSSÃO}

Na Odontologia, a ética desperta máxima atenção não só pelo crescente número de profissionais formados na área, mas também pela concorrência elevada no mercado de trabalho. No marketing odontológico, o controle de publicidade se dá através de dois dispositivos: o Código de Defesa do Consumidor (BRASIL, 1990) e o CEO, que, em seu Capítulo XVI, estabelece padrões normativos para as publicidades em Odontologia, visando estabelecer condições iguais para todos os profissionais (BRASIL, 2012; GARBIN et al., 2010; SILVA et al., 2013).

Com base na presente pesquisa, observou-se que a maior parte dos estabelecimentos onde os serviços odontológicos são prestados é caracterizada como consultório (70,2\%). De acordo com a literatura, consultórios são os estabelecimentos com 1 (um) único conjunto de equipamentos odontológicos, enquanto clínicas são caracterizadas por um conjunto de consultórios odontológicos independentes entre si (DA COSTA, 2015; RIO GRANDE DO SUL, 2000). Neste estudo, observou-se a predominância de consultórios odontológicos em detrimento de clínicas. Atribui-se a esta realidade o alto custo dos equipamentos odontológicos, que torna menos oneroso montar consultórios do que clínicas, bem como o desejo de muitos jovens profissionais de trabalharem em consultório privado (FERREIRA; FERREIRA; FREIRE, 2013). Gomes e Ramos (2015) justificam esse fato pela tradição do liberalismo profissional que caracteriza a Odontologia, tornando inerente ao profissional o desejo de possuir o próprio consultório.

A maioria das pessoas procura serviços odontológicos próximos de suas residências a fim de evitar grandes deslocamentos e fadiga (MORANO JÚNIOR; MIALHE, 2007). Porém, este estudo verificou uma expressiva aglomeração de estabelecimentos no centro da cidade $(49,1 \%)$, diferente do apontado por Morano Júnior e Mialhe (2007) e dos achados de Martins et al. (2011), que observaram uma prevalência relativamente baixa nessa região $(21,4 \%)$. As prováveis causas da preferência dos profissionais de Campina Grande pelo centro da cidade podem estar relacionadas ao maior fluxo de pessoas e potenciais pacientes que essa área fornece; à oferta de transporte público que facilita o acesso de pacientes de áreas mais afastadas; ou, até mesmo, ao fato de os consultórios e clínicas estarem próximos das lojas fornecedoras de materiais de consumo e instrumentais, assim como de laboratórios prestadores de serviços. Essa aglomeração de profissionais na mesma área acirra, ainda mais, a competitividade profissional e, em contrapartida, torna escassa a oferta de cirurgiões-dentistas nos demais bairros.

Constatou-se que o principal veículo de comunicação externa utilizado pelos cirurgiões-dentistas é a placa $(84,2 \%)$, dado semelhante ao reportado por Garbin et al. (2010) e Martins et al. (2011), que encontraram uma frequência de $71,9 \%$ e $83,4 \%$, respectivamente. Esse formato de mídia externa tem por objetivo informar à sociedade sobre a presença do profissional e de suas qualificações, podendo ser feito de diversos materiais (GARBIN et al., 2010), apresentando custo relativamente baixo e facilmente encontrado no mercado.

Neste estudo, foi possível verificar que 35,1\% dos consultórios e 19,3\% das clínicas informaram suas áreas de atuação. Entretanto, não cumpriram a normatização imposta pelo CEO, já que apenas $1,8 \%$ de cada uma das categorias incluíram o título de especialista, e nenhum estabelecimento apresentou a expressão "clínico geral"; o que corrobora com os achados de Martins et al. (2011), os quais observaram que $75,7 \%$ dos anúncios não apresentaram a qualificação profissional de "clínico geral".

Dentre as áreas de atuação analisadas nos anúncios e placas, as mais prevalentes foram a Reabilitação Oral/Prótese (33,3\%), Dentística (32,1\%) e Ortodontia (23,2\%). No trabalho executado por Garbin et al. (2010), a especialidade mais prevalente foi a Ortodontia $(62,7 \%)$, seguida pela Implantodontia (40,3\%) e Reabilitação Oral/ Prótese (38,8\%). Paranhos (2009) aponta a Ortodontia, Implantodontia e Prótese Dentária como especialidades mais registradas nos estados da região Centro-Oeste. Esta escolha pode ser atribuída ao mercado promissor que tais especialidades oferecem no que se refere à demanda de pacientes, visto que muitos desses serviços não são 
oferecidos pelo Sistema Único de Saúde (SUS), além do fato de ser bastante significativo o volume financeiro que estes tratamentos podem oferecer ao profissional.

A capacitação e atualização são fundamentais para a atuação no mercado odontológico, constituindo-se em um dever para o cirurgião-dentista segundo o Código de Ética Odontológico (BRASIL, 2012). Apesar de os cirurgiões-dentistas estarem entre os profissionais que mais se qualificam através de cursos de especialização e capacitação (TOMAZ, 2011), constatou-se, neste estudo, que apenas uma pequena porcentagem de profissionais possuía o título de especialista descrito nos anúncios de publicidade. Infere-se que a ausência de qualificação, dentre outros motivos, possa ser atribuída ao alto investimento necessário para a realização de um curso de especialização.

o presente estudo verificou que apenas $12,3 \%$ dos consultórios e clínicas possuíam todos os itens obrigatórios, semelhante a outros dois estudos realizados na região nordeste, precisamente nos municípios de São Luís/MA (2\%) (MARTINS et al., 2011) e Jequié/BA (11,76\%) (SILVA et al., 2013) com prevalências inferiores. Porém, é salutar apontar que, em uma pesquisa, realizada em São Paulo-SP, observou-se uma elevada taxa de consultórios e clínicas que seguiam as normas quanto aos itens obrigatórios determinados pelo CEO $(44,9 \%)$ (GARBIN et al., 2010). Essa desigualdade pode estar relacionada com 0 tipo de mercado, ou com a postura do Conselho Regional de Odontologia de cada estado da federação, que tem por obrigação fiscalizar e punir essas irregularidades (BRASIL, 2012), uma vez que essas informações estão visíveis e podem ser facilmente constatadas.

Um fato que deve ser destacado no presente estudo é a substituição do nome "cirurgião-dentista", representativo da profissão, por termos populares como: dentista, odontologia, "odonto", consultório odontológico, a própria especialidade odontológica, ou por alguma imagem relacionada à Odontologia, o que foi verificado em $86,0 \%$ da amostra. Garbin et al. (2010) enfatizaram que tais expressões, indiscutivelmente, não possuem o mesmo valor, apontando não só infração ética por não apresentar o item obrigatório, mas também por demonstrar desvalorização pela própria profissão perante a sociedade.

Nesta pesquisa, verificou-se a troca de termos que se referem às especialidades por termos coloquiais, como: "canal" ao invés de Endodontia; e do termo "reabilitação" que é bastante abrangente e pode fazer alusão à Dentística Restauradora, à Prótese Dentária e ainda a tratamentos de Articulação Têmporo Mandibular. Segundo Garbin et al. (2010), o uso de termos coloquiais facilita a comunicação com a população que não apresenta o mesmo conhecimento científico do profissional.

Dos itens permitidos pelo CEO para aparecer nas placas e anúncios, o mais prevalente foi o número do telefone $(77,2 \%)$, semelhante ao descrito por Silva et al. (2013) (52,94\%), Garbin et al. (2010) $(65,2 \%)$ e Martins et al. (2011) $(85,4 \%)$, demonstrando que este é o principal meio de comunicação paciente-profissional-paciente.
Destaca-se, nesta pesquisa, o fato de que 5,3\% dos estabelecimentos apresentarem algum item proibido pelo CEO, constituindo infração ética. As infrações mais prevalentes foram: apresentação de imagens de "antes e depois" $(1,8 \%)$, que caracteriza a Odontologia como ofício de obrigação de resultados (TERADA; GALO; SILVA, 2014); e modalidade de pagamento ( $5,3 \%$ ), que, de acordo com o CEO (2012), confere imagem de mercantilização em um ambiente que deve ser de promoção da saúde. Entretanto, Tomaz (2011) concebe o consultório odontológico como uma empresa moderna, e aponta a necessidade de flexibilização do CEO para garantir melhores condições para a prática liberal da Odontologia. Porém, essas infrações caracterizam conduta inadequada e devem ser coibidas por parte dos Conselhos Regionais de Odontologia através de fiscalização contínua e campanhas de conscientização constantes.

Segundo Viola, Oliveira e Dotta (2011), é imprescindível a conscientização do profissional quanto à responsabilidade sobre qualquer anúncio veiculado. Em um estudo sobre processos éticos no CRO de Minas Gerais, Bouchardet et al. (2016) apontaram que 30,4\% desses processos foram instaurados devido a irregularidades nas propagandas. Em pesquisa semelhante, observou-se que $33,9 \%$ dos processos tinham como razão a propaganda irregular (GIFFONI FILHO et al., 2013).

Apenas 12,3\% dos consultórios e clínicas apresentam-se em total consonância com as normas do Capítulo XVI, que trata exclusivamente das formas de divulgação da Resolução CFO-118/2012 do CEO, o que pode ser considerado um número muito baixo. Neste aspecto, ganham importância as instituições que formam os profissionais da Odontologia, que devem informar sobre a responsabilidade social do cirurgião-dentista, realizando, ainda durante a graduação, atividades voltadas ao pensamento crítico e à conscientização sobre aspectos éticos.

Algumas limitações devem ser destacadas, como, por exemplo, a amostragem intencional e ausência de cálculo amostral para determinação do quantitativo mínimo de profissionais e clínicas dentárias em função da não cessão da lista de inscritos no CRO-PB. Todavia, a despeito desse fato, os resultados obtidos são expressivos, pois retratam um tema pouco estudado em Odontologia, contribuindo, portanto, para a sua melhor compreensão.

Face ao exposto, observa-se a necessidade de ações urgentes por parte dos órgãos de classe e das instituições de ensino superior, na tentativa de trabalhar a conscientização em cumprir a legislação da profissão, coibir as infrações já existentes e de prevenir outras que possam vir a acontecer devido à concorrência cada vez maior no mercado de trabalho.

\section{CONCLUSÕES}

De acordo com os parâmetros estabelecidos pelo Código de Ética Odontológico quanto à publicidade e propaganda, conclui-se que uma pequena porcentagem dos 
cirurgiões-dentistas e clínicas odontológicas analisados no presente estudo está seguindo as normas estabelecidas pelo referido código; e, dentre os itens terminantemente proibidos, encontra-se a modalidade de pagamento como infração mais prevalente.

\section{REFERÊNCIAS}

BENEDICTO, E. N. et al. Principais alterações do novo código de ética odontológica brasileiro de 2012. Salusvita, Bauru, v. 32, n. 2, p. 149161, 2013.

BOUCHARDET, F. C. H. et al. Ethical Proceedings Involving Dentists In The State Of Minas Gerais, Brazil. RBOL, Ribeirão Preto, v. 3, n. 1, p. 24-31, 2016.

BRASIL. Lei no 8.078, de 11 de setembro de 1990. Código de Defesa do Consumidor. Brasília, 1990.

BRASIL. Conselho Federal de Odontologia. Resolução CFO n ${ }^{\circ} 118$, de 11 de maio de 2012. Revoga o Código de Ética Odontológica aprovado pela resolução CFO-42/2003 e aprova outro em substituição.Diário Oficial [da] União, Brasília, DF, 14 jun. 2012.

CAVALCANTI, A. L. et al. Odontologia e o Código de Defesa do Consumidor: análise dos processos instaurados contra cirurgiões-dentistas e planos odontológicos em Campina Grande - Paraíba. Rev. odontol. UNESP. Marilia, v. 40, n. 1, p. 6-11, 2011.

CONSELHO FEDERAL DE ODONTOLOGIA (CFO). Faculdades. Lista de Faculdades de Odontologia por Estado. 2009-2015. Disponível em: < http:// cfo.org.br/servicos-e-consultas/faculdades/>. Acesso em: 10 maio 2016.

DA COSTA, L. A. Odontologia e Modernidade: relatos de vida profissional de odontólogos brasileiros. Rev. Inter. Humanid. Méd. Madrid, v. 4, n. 2, p. 125-132, 2015.

FERREIRA, N. P; FERREIRA, A. P; FREIRE, M. C. M. Job market in dentistry: historical context and perspectives. Rev. odontol. UNESP. Marília, v. 42, n. 4, p. 304-309, 2013.

GARBIN, A. J. I. et al. Publicidade em odontologia : avaliação dos aspectos éticos envolvidos. RGO, Porto Alegre, v. 58, n. 1, p. 85-89, 2010.

GIFFONI FILHO, R. I. R. et al. Labor disputes on grounds of discipline in a Brazilian regional concil of dentistry: a critic analysis. RGO, Porto Alegre, v. 61 , n. 2, p. 245-253, 2013.

GOMES, D.; RAMOS, F. R. S. The dental professional after productive restructuring: ethics, the job market and dental public health'. Saúde Soc., São Paulo, v. 24, n. 1, p. 285-297, 2015.

INSTITUTO BRASILEIRO DE GEOGRAFA E ESTATÍSTICA (IBGE). Cidades. Campina Grande, 2016. Disponível em: < http://cod.ibge.gov.br/1V6
>. Acesso em: 10 maio 2016.

INSTITUTO NACIONAL DE ESTUDOS E PESQUISAS EDUCACIONAIS ANÍSIO TEIXEIRA (INEP). Censo da educação superior 2013: resumo técnico. 2015. Disponível em: 〈http://goo.gl/7P4i1s>. Acesso em: 10 maio 2016.

MARTINS, A. L. G. et al. Assessment of ethical aspects of advertising and propaganda disseminated by dental professionals in São Luís - Maranhão. J. health res., São Luís, v. 12, n. 2, p. 23-26, 2011.

MORANO JÚNIOR, M.; MIALHE, F.L. Critérios utilizados por diferentes grupos etários na escolha do cirurgião-dentista. Odontol. clín.-cient., Recife, v. 6, n. 1, p. 33-37, 2007.

PACHECO, K.T.S.; SILVA JÚNIOR, M. F.; MEIRELES, N. R. Ethical proceedings against dentists in Espírito Santo for infringements to the code of dental ethics. Braz. oral res., São Paulo, v. 28, n. 1, p. 1-7, 2014.

PARAÍBA. Lei Complementar no 92, de 11 de dezembro de 2009. Institui a região metropolitana de Campina Grande e dá outras providências. Diário Oficial do Estado [da] Bahia. João Pessoa, PB, 11 dez. 2009.

PARANHOS, L.R. et al. An analysis of the dentistry job market in the Center-west region of Brazil. ROBRAC, Goiânia, v. 18, n. 45, p. 48-55, 2009.

PARANHOS, L.R. et al. Implicações éticas e legais do marketing na Odontologia. RSBO, Joinville, v. 8, n. 2, p. 219-224, 2011.

RIO GRANDE DO SUL. Secretaria de Estado da Saúde do Rio Grande do Sul (SES-RS). Norma técnica de biossegurança em estabelecimentos odontológicos e laboratórios de prótese dentária no RS. 2000. Disponível em: < http://www1.saude.rs.gov.br/dados/1286567156896scr11\%20 portaria\%2040\%202000\%20normas\%20bioseguranca\%20odontologicos.pdf >. Acesso em: 10 maio 2016.

SILVA, I.S.N. et al. Assessment of the ethical aspects of boards dentist in Jequié - BA. Rev. saúde. com, Jequié, v. 9, n. 1, p. 44-50, 2013.

TELELISTAS. NET. Telelistas.net. 2016. Disponível em: < http://www. telelistas.net/templates/resultado_busca.aspx?q=dentista\%2BCampin $a+$ Grande\&orgm $=0 \&$ cod_localidade $=83040$ \&atividade $=$ dentista\&nome =\&uf_busca=pb\&image.x=70\&image.y=11>. Acesso em: 10 maio 2016 .

TERADA, A. S. S. D.; GALO, R.; SILVA, R. H. A. Civil liability of the dental surgeon: professionals' knowledge. Arq. Odontol., Belo Horizonte, v. 50, n. 2, p. 92-97, 2014.

TOMAZ, P. A. R. A nova visão da Odontologia. In: TOMAZ, P. A. R. Marketing para dentistas: orientações ao consultório-empresa. 5. ed. São Paulo: Navegar, 2011. cap. 1, p. 19.

VIOLA, N. V.; OLIVEIRA, A. C. M.; DOTTA, E. A. V. Marketing in dentistry: a tool that makes the difference. Rev. bras. odontol., Rio de Janeiro, $v$. 68, n. 2, p. 248-251, 2011.

Submetido em: 16/09/2016

Aceito em: 10/04/2017 